COMMENT. Biochemical and ultrastructural abnormalities indicative of mitochondrial disease are sufficiently frequent to recommend muscle biopsy as an important diagnostic examination in children with unexplained mental retardation. Both ultrastructural mitochondrial abnormalities and decreased activity of 1 or more respiratory chain enzymes are required for a probable diagnosis of mitochondrial disease. The commonly known mtDNA mutations are a rare cause of childhood encephalomyopathies, in contrast to the adult form that frequently shows the MELAS mutation.

Mitochondrial DNA (mtDNA) defects in neuromuscular disorders are reviewed by Marin-Garcia J. and Goldenthal MJ (Pediatr Neurol February 2000;22:122-129) at the Molecular Cardiology Institute, Highland Park, NJ. Mitochondrial mtDNA deletions were found in 1 child with Kearns-Sayre disease, 1 with stroke/CADASIL, and 1 with progressive external ophthalmoplegia, hypotonia, developmental delay, and lactic acidosis. Reduced mtDNA levels occurred in 2 children with encephalomyopathy, hypotonia, lactic acidosis, and mtDNA depletion. Pathogenic mtDNA point mutations are maternally inherited, and most are located in tRNA and rRNA genes.

\title{
CLINICAL APPROACH TO METABOLIC MYOPATHIES
}

The clinical and laboratory evaluation of the patient with suspected metabolic myopathy is reviewed from the Department of Neurology, Children's Hospital, Boston, MA. Myopathies are classified as static characterized by proximal weakness, generalized weakness, and developmental delay; and dynamic with recurrent episodes of reversible muscle dysfunction, sometimes myoglobinuria, related to exercise intolerance, fasting, exposure to cold, anesthesia, intercurrent infection, or a low-carbohydrate, high-fat diet. Both forms are common in mitochondrial myopathies. The type or duration of exercise inducing weakness may be specific: Prolonged, low-intensity activity (eg walking) - induced weakness occurs with fatty acid oxidation (FAO) defects; high-intensity exercise (eg weight lifting or sprinting) - glycogen or glucose metabolism defects. Myoglobinuria may be induced by inborn errors of glycogen/glucose metabolism, FA metabolism, and some mitochondrial cytopathies. Laboratory tests include CK, elevated in glycogen defects and lactate dehydrogenase deficiency; blood lactate and pyruvate elevated in mitochondrial myopathies; liver transaminases elevated in FAO defects; and abnormal carnitine, acylcarnitine, free fatty acids, and hypoketotic hypoglycemia in lipid metabolic disorders. EMG, Forearm Ischemic Exercise Test, and muscle biopsy with molecular studies may be required in diagnosis. (Darras BT, Friedman NR. Metabolic myopathies: a clinical approach; Part I. Pediatr Neurol February 2000;22:87-97). (Respond: Dr Darras, Neuromuscular Program, Neurology Department, Fegan 11, Children's Hospital, 300 Longwood Avenue, Boston, MA 02115).

COMMENT. A helpful algorithm for the step-by-step diagnosis of metabolic myopathies is provided by the authors.

\section{SEIZURE DISORDERS}

\section{GELASTIC EPILEPSY AND HYPOTHALAMIC HAMARTOMA}

Three patients with small hypothalamic hamartomas and a recurrent "pressure to laugh," often without actual laughter, are reported from the University of Melbourne, Australia, and McGill University, Canada. Giggling 